American Journal of Applied Sciences 8 (3): 254-260, 2011

ISSN 1546-9239

(C) 2010 Science Publications

\title{
Thermal Efficiency of Double Pass Solar Collector with Longitudinal Fins Absorbers
}

\author{
Ahmad Fudholi, Kamaruzzaman Sopian, Mohd Hafidz Ruslan, \\ Mohd. Yusof Othman and Muhammad Yahya \\ Solar Energy Research Institute, University Kebangsaan Malaysia, \\ 43600 UKM Bangi, Selangor Darul Ehsan, Malaysia
}

\begin{abstract}
Problem statement: One of the most important components of a solar energy system is the solar collector. The performances of double-pass solar collector with longitudinal fins absorbers are analyzed. Approach: The study involves a theoretical study to investigate the effect of mass flow rate, number and height of fins on efficiency, which involves steady-state energy balance equations on the longitudinal fins absorber of solar collectors. The theoretical solution procedure of the energy equations uses a matrix inversion method and making some algebraic rearrangements. Results: The collector efficiency increases as the number and height of fins increases. For a mass flow rate 0.02$0.1 \mathrm{~kg} / \mathrm{s}$, the double-pass solar collectors are efficiency about $36-73 \%$ in upper fins (type I), $37-75 \%$ in lower fins (type II) and 46-74\% in upper and lower fins (type III). Conclusion: The efficiency of the collector is strongly dependent on the flow rate, efficiency increase is about $35 \%$.
\end{abstract}

Key words: Double pass solar collector, fins absorbers, thermal efficiency, solar collector

\section{INTRODUCTION}

Solar air collectors are simple, cheap and most widely used. It used primarily for space heating, solar desalination, solar water heater, industrial process heat and also for drying. Solar energy includes system of drying is very attractive application and cost competitive such as drying of cocoa, coffee beans, fruits, noodle, rubber, or marine products. (Fudholi et al., 2010; Ebrahim et al., 2009). Conventional solar air collectors have inherent disadvantages is lower thermal efficiency. Various designs of solar collectors have been the subject of many theoretical and experimental investigations. Helal et al. (2010) studied energetic performances of an integrated collector storage solar water heater. The systems shows little cost, simplicity and simpler to be installed on the building roof. Prasad et al. (2010) studied experiment analysis of flat plate collector and comparison of performance with tracking collector. Dammak et al. (2010) optimized hybrid of flat plate collector with a bubble pump for absorptiondiffusion cooling systems. Reda (2010) studied the stability of luminescent solar collector prepared by solgel spin coating method using Ponceau 2R.

There are many design alternatives to the conventional single pass collector. These designs must be able to reduce the heat losses from the collector resulting in the increase in the operating temperatures of the system and higher collector efficiencies. Therefore, single pass solar collector with porous media has been introduced, inexpensive porous materials such as stones, crushed glass, wool plants and metal wool. The multi pass solar collector with advanced heat transfer enhancement features such as fins and porous media have better performance than the conventional single pass collector. Sopian et al. (2009) studied the effects of changes in upper and lower channel depth on the thermal efficiency with and without porous media of the double-pass solar air collector for various operation conditions. They concluded that typical thermal efficiency of the double-pass solar air collector with porous media is about $60-70 \%$. The addition of the porous media in the second channel of the double-pass solar collector increases the performance of the collector. The theoretical model has been developed and the experimental validation has been carried out. It was shown that the theoretical simulation and experimental data were in close agreement with each other. Introducing porous media in the second channel increases the heat transfer area. Pradhapraj et al. (2010) reviewed on porous and non porous flat plate air collector with mirror enclosure. They discussed the performances of porous and non-porous absorber plates, the possible methods of finding out air leakages and the methodology adopted for the performance and efficiency calculations.

The enhancement of corrugated surfaces to use an extended heat transfer of solar collectors has been investigated, such as by using matrix type absorber, compound honeycomb collector, box-type absorber and 


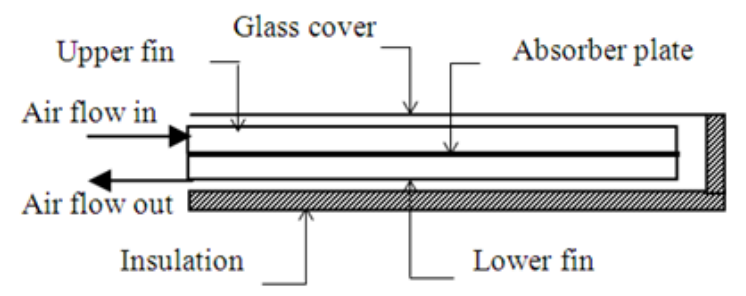

Fig. 1: Schematic of the double pass solar collector with longitudinal

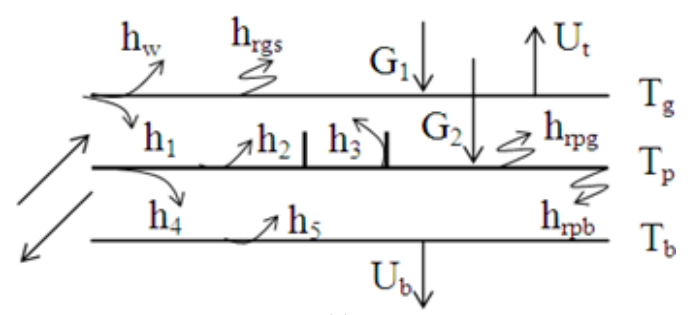

(a)

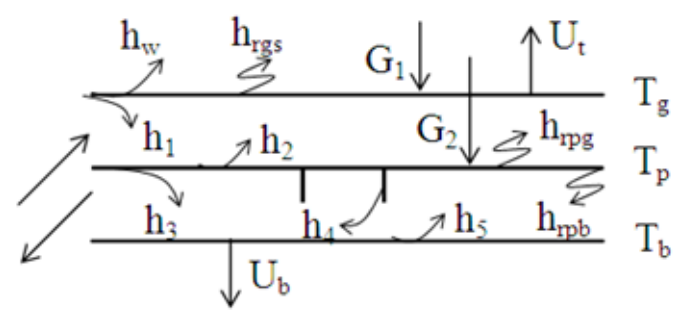

(b)

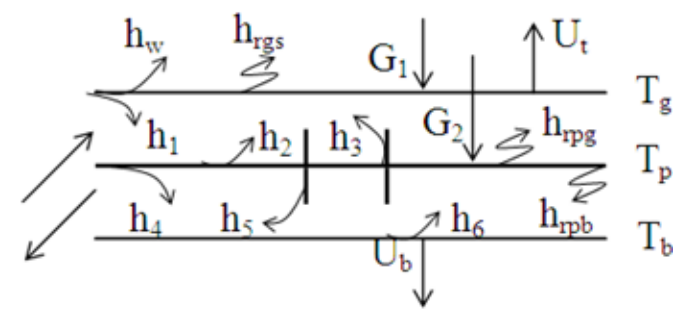

(c)

Fig. 2: Schematic of heat transfer coefficients in double pass solar collector, (a) upper fin (type I), (b) lower fin (type II) and (c) upper and lower fin (type III)

finned absorber. In the present study, the main concern is to study theoretically to investigate the effect of mass flow rate, number and height of fins on efficiency of double pass solar collector.

Theoretical analysis: Figure1 shows the configuration of double pass solar collector with longitudinal fins.

Figure 2 shows the various heat transfer coefficients of three types double pass solar collectors with longitudinal fin absorbers. Figure 3 shows energy balance for each element of the fin with a height $(\mathrm{dz})$.

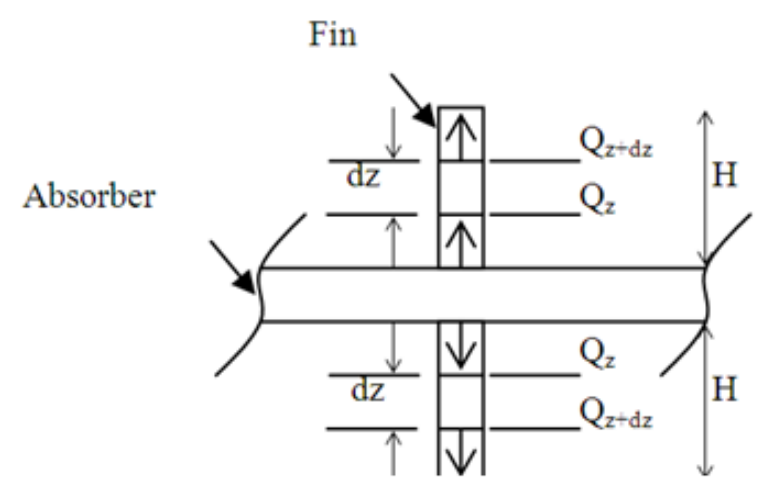

Fig. 3: Schematic of energy balance for each element of the fin

\section{MATERIALS AND METHODS}

To simplify the analysis, the following assumptions have been made (a) performance is steady state (b) all convection heat transfer coefficients in channels and flowing air are equal and constant (3) thermal conductivity of fin and absorber are constant and (4) the useful heat gain to the air is uniform along the length of the collector.

The steady state energy balance equations for typeI (upper fin) can be written as follow:

$T_{g}: U_{t}\left(T_{g}-T_{a}\right)+h_{1}\left(T_{g}-T_{f 1}\right)=h_{r p g}\left(T_{p}-T_{g}\right)+\alpha_{g} I$

$\mathrm{T}_{\mathrm{fl}}: \mathrm{Q}_{1}=\mathrm{h}_{1}\left(\mathrm{~T}_{\mathrm{g}}-\mathrm{T}_{\mathrm{f} 1}\right)+\mathrm{h}_{2}\left(\mathrm{~T}_{\mathrm{p}}-\mathrm{T}_{\mathrm{f} 1}\right)+\frac{\mathrm{N}}{\mathrm{A}_{\mathrm{f}}} \mathrm{Q}_{\mathrm{fn} 1}$

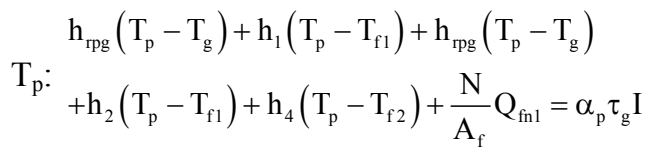

$\mathrm{T}_{\mathrm{f} 2}: \mathrm{Q}_{2}=\mathrm{h}_{4}\left(\mathrm{~T}_{\mathrm{p}}-\mathrm{T}_{\mathrm{f} 2}\right)+\mathrm{h}_{5}\left(\mathrm{~T}_{\mathrm{b}}-\mathrm{T}_{\mathrm{f} 2}\right)$

$\mathrm{T}_{\mathrm{b}}: \mathrm{h}_{\mathrm{rpb}}\left(\mathrm{T}_{\mathrm{p}}-\mathrm{T}_{\mathrm{b}}\right)=\mathrm{h}_{5}\left(\mathrm{~T}_{\mathrm{b}}-\mathrm{T}_{\mathrm{f} 2}\right)+\mathrm{U}_{\mathrm{b}}\left(\mathrm{T}_{\mathrm{b}}-\mathrm{T}_{\mathrm{a}}\right)$

For type II (lower fin):

$T_{g}: U_{t}\left(T_{g}-T_{a}\right)+h_{1}\left(T_{g}-T_{f 1}\right)=h_{r p g}\left(T_{p}-T_{g}\right)+\alpha_{g} I$

$\mathrm{T}_{\mathrm{f} 1}: \mathrm{Q}_{1}=\mathrm{h}_{1}\left(\mathrm{~T}_{\mathrm{g}}-\mathrm{T}_{\mathrm{fl}}\right)+\mathrm{h}_{2}\left(\mathrm{~T}_{\mathrm{p}}-\mathrm{T}_{\mathrm{f} 1}\right)$

$$
\mathrm{T}_{\mathrm{p}}: \begin{aligned}
& \mathrm{h}_{\mathrm{rpg}}\left(\mathrm{T}_{\mathrm{p}}-\mathrm{T}_{\mathrm{g}}\right)+\mathrm{h}_{2}\left(\mathrm{~T}_{\mathrm{p}}-\mathrm{T}_{\mathrm{f} 1}\right)+\mathrm{h}_{\mathrm{rpg}}\left(\mathrm{T}_{\mathrm{p}}-\mathrm{T}_{\mathrm{g}}\right) \\
& +\mathrm{h}_{3}\left(\mathrm{~T}_{\mathrm{p}}-\mathrm{T}_{\mathrm{f} 2}\right)+\frac{\mathrm{N}}{\mathrm{A}_{\mathrm{f}}} \mathrm{Q}_{\mathrm{fn} 2}=\alpha_{\mathrm{p}} \tau_{\mathrm{g}} \mathrm{I}
\end{aligned}
$$


$\mathrm{T}_{\mathrm{f} 2}: \mathrm{Q}_{2}=\mathrm{h}_{3}\left(\mathrm{~T}_{\mathrm{p}}-\mathrm{T}_{\mathrm{f} 2}\right)+\mathrm{h}_{5}\left(\mathrm{~T}_{\mathrm{b}}-\mathrm{T}_{\mathrm{f} 2}\right)+\frac{\mathrm{N}}{\mathrm{A}_{\mathrm{f}}} \mathrm{Q}_{\mathrm{fn} 2}$

$T_{b}: h_{r p b}\left(T_{p}-T_{b}\right)=h_{5}\left(T_{b}-T_{f 2}\right)+U_{b}\left(T_{b}-T_{a}\right)$

For type III (upper and lower fin):

$T_{g}: U_{t}\left(T_{g}-T_{a}\right)+h_{1}\left(T_{g}-T_{f 1}\right)=h_{r g g}\left(T_{p}-T_{g}\right)+\alpha_{g} I$

$T_{f 1}: Q_{1}=h_{1}\left(T_{g}-T_{f 1}\right)+h_{2}\left(T_{p}-T_{f 1}\right)+\frac{N}{A_{f}} Q_{f n 1}$

$\begin{aligned} T_{p}: & h_{\text {rpg }}\left(T_{p}-T_{g}\right)+h_{2}\left(T_{p}-T_{f 1}\right)+h_{r p g}\left(T_{p}-T_{g}\right) \\ & h_{4}\left(T_{p}-T_{f 2}\right)+\frac{N}{A_{f}} Q_{f i 1}+\frac{N}{A_{f}} Q_{f n 2}=\alpha_{p} \tau_{g} I\end{aligned}$

$T_{\mathrm{f} 2}: \mathrm{Q}_{2}=\mathrm{h}_{4}\left(\mathrm{~T}_{\mathrm{p}}-\mathrm{T}_{\mathrm{f} 2}\right)+\mathrm{h}_{6}\left(\mathrm{~T}_{\mathrm{b}}-\mathrm{T}_{\mathrm{f} 2}\right)+\frac{\mathrm{N}}{\mathrm{A}_{\mathrm{f}}} \mathrm{Q}_{\mathrm{fn} 2}$

$T_{b}: h_{r p b}\left(T_{p}-T_{b}\right)=h_{6}\left(T_{b}-T_{f 2}\right)+U_{b}\left(T_{b}-T_{a}\right)$

Where:

$\mathrm{Q}_{1}=2 \dot{\mathrm{m} C}\left(\mathrm{~T}_{\mathrm{f} 1}-\mathrm{T}_{\mathrm{fl}, \mathrm{i}}\right) / \mathrm{wL}$

$\mathrm{Q}_{2}=2 \dot{\mathrm{m} C}\left(\mathrm{~T}_{\mathrm{f} 2}-\mathrm{T}_{\mathrm{f} 2, \mathrm{i}}\right) / \mathrm{wL}$

$\mathrm{Q}_{\mathrm{fn} 1}=\mathrm{Q}_{\mathrm{z}=0}=-\mathrm{kA}_{\mathrm{sf}}\left(\frac{\mathrm{dT}_{\mathrm{ffl} 1}}{\mathrm{dz}}\right)_{\mathrm{z}=0}$

$=\left(2 \mathrm{k}_{\mathrm{p}} \mathrm{A}_{\mathrm{sf}} \mathrm{L}_{\mathrm{ffl}}\right)^{1 / 2}\left(\mathrm{~T}_{\mathrm{p}}-\mathrm{T}_{\mathrm{fl}}\right) \tanh \mathrm{MH}$

$\mathrm{Q}_{\mathrm{fn} 2}=-\mathrm{kAsf}\left(\frac{\mathrm{dT}_{\mathrm{fn} 2}}{\mathrm{dz}}\right)_{\mathrm{z}=0}$

$=\left(2 \mathrm{k}_{\mathrm{p}} \mathrm{A}_{\mathrm{sf}} \mathrm{Lh}_{\mathrm{fn} 2}\right)^{1 / 2}\left(\mathrm{~T}_{\mathrm{p}}-\mathrm{T}_{\mathrm{f} 2}\right) \tanh \mathrm{MH}$

Theoretical solution procedure: The theoretical model assumes that for a short collector, the temperatures of the wall surrounding the airflow are uniform and temperatures of the airflow vary linearly along the collector. For the short collectors, the mean air temperature is then equal to the arithmetic mean (Ong, 1995).

Where:

$$
\begin{aligned}
& T_{f 1}=\left(T_{f 1, o}+T_{i}\right) / 2 \\
& T_{f 2}=\left(T_{f 2, o}+T_{f 1, o}\right) / 2
\end{aligned}
$$

In general, the above Eq. 1-15 can be presented in a $5 \times 5$ matrix form. The above matrices may be displayed as:

$[\mathrm{A}][\mathrm{T}]=[\mathrm{B}]$

For type I:

$\left[\begin{array}{ccccc}\mathrm{S}_{6} & -\mathrm{h}_{1} & -\mathrm{h}_{\mathrm{rpg}} & 0 & 0 \\ \mathrm{~h}_{1} & \mathrm{~S}_{7} & \mathrm{~h}_{2} & 0 & 0 \\ -\mathrm{h}_{\mathrm{rpg}} & -\mathrm{h}_{2} & \mathrm{~S}_{8} & \mathrm{~S}_{9} & -\mathrm{h}_{\mathrm{rpb}} \\ 0 & 4 \dot{\mathrm{mC}} / \mathrm{wL} & \mathrm{S}_{10} & \mathrm{~S}_{11} & \mathrm{~h}_{5} \\ 0 & 0 & \mathrm{~h}_{\mathrm{rpb}} & \mathrm{h}_{5} & \mathrm{~S}_{12}\end{array}\right]\left[\begin{array}{c}\mathrm{T}_{\mathrm{g}} \\ \mathrm{T}_{\mathrm{f} 1} \\ \mathrm{~T}_{\mathrm{p}} \\ \mathrm{T}_{\mathrm{f} 2} \\ \mathrm{~T}_{\mathrm{b}}\end{array}\right]=\left[\begin{array}{c}\mathrm{S}_{1} \\ \mathrm{~S}_{2} \\ \mathrm{~S}_{3} \\ \mathrm{~S}_{4} \\ \mathrm{~S}_{5}\end{array}\right]$

Where:

$\mathrm{S}_{1}=\mathrm{U}_{\mathrm{t}} \mathrm{T}_{\mathrm{a}}+\alpha_{\mathrm{g}} \mathrm{I}$

$\mathrm{S}_{2}=-(2 \dot{\mathrm{m} C} / \mathrm{wL}) \mathrm{T}_{\mathrm{i}}$

$\mathrm{S}_{3}=\alpha_{\mathrm{p}} \tau_{\mathrm{g}} \mathrm{I}$

$\mathrm{S}_{4}=-\mathrm{S}_{2}$

$\mathrm{S}_{5}=-\mathrm{T}_{\mathrm{a}} \mathrm{U}_{\mathrm{b}}$

$\mathrm{S}_{6}=\mathrm{h}_{1}+\mathrm{h}_{\mathrm{rpg}}+\mathrm{U}_{\mathrm{t}}$

$\mathrm{S}_{7}=-\left[\begin{array}{l}\mathrm{h}_{1}+\mathrm{h}_{2}+(2 \mathrm{mC} / \mathrm{wL}) \\ +\frac{\mathrm{N}}{\mathrm{A}_{\mathrm{f}}}\left(2 \mathrm{kA}_{\mathrm{sf}} \mathrm{Lh}_{3}\right)^{1 / 2} \tanh \mathrm{MH}\end{array}\right]$

$\mathrm{S}_{8}=\mathrm{h}_{2}+\frac{\mathrm{N}}{\mathrm{A}_{\mathrm{f}}}\left(2 \mathrm{kA}_{\mathrm{sf}} \mathrm{Lh}_{3}\right)^{1 / 2} \tanh \mathrm{MH}$

$\mathrm{S}_{9}=-\mathrm{S}_{8}$

$\mathrm{S}_{10}=\mathrm{h}_{2}+\mathrm{h}_{4}+\mathrm{h}_{\mathrm{rpg}}+\mathrm{h}_{\mathrm{rpb}}+\frac{\mathrm{N}}{\mathrm{A}_{\mathrm{f}}}\left(2 \mathrm{kA}_{\mathrm{sf}} \mathrm{Lh}_{3}\right)^{1 / 2} \tanh \mathrm{MH}$

$\mathrm{S}_{11}=-\left[\mathrm{h}_{4}+\mathrm{h}_{5}+(2 \dot{\mathrm{m} C} / \mathrm{wL})\right]$

$\mathrm{S}_{12}=-\left(\mathrm{h}_{5}+\mathrm{h}_{\mathrm{rpb}}+\mathrm{U}_{\mathrm{b}}\right)$

$\mathrm{M}=\left(\frac{2 \mathrm{Lh}_{3}}{\mathrm{k}_{\mathrm{p}} \mathrm{A}_{\mathrm{sf}}}\right)^{1 / 2}$

For type II: 
Am. J. Applied Sci., 8 (3): 254-260, 2011

$\left[\begin{array}{ccccc}\mathrm{S}_{6} & -\mathrm{h}_{1} & -\mathrm{h}_{\mathrm{rpg}} & 0 & 0 \\ \mathrm{~h}_{1} & \mathrm{~S}_{7} & \mathrm{~S}_{8} & 0 & 0 \\ -\mathrm{h}_{\mathrm{rpg}} & \mathrm{S}_{9} & \mathrm{~S}_{10} & -\mathrm{h}_{4} & -\mathrm{h}_{\mathrm{rpb}} \\ 0 & 4 \dot{\mathrm{mC}} / \mathrm{wL} & \mathrm{h}_{4} & \mathrm{~S}_{11} & \mathrm{~h}_{5} \\ 0 & 0 & \mathrm{~h}_{\mathrm{rpb}} & \mathrm{h}_{5} & \mathrm{~S}_{12}\end{array}\right]\left[\begin{array}{c}\mathrm{T}_{\mathrm{g}} \\ \mathrm{T}_{\mathrm{f} 1} \\ \mathrm{~T}_{\mathrm{p}} \\ \mathrm{T}_{\mathrm{f} 2} \\ \mathrm{~T}_{\mathrm{b}}\end{array}\right]=\left[\begin{array}{c}\mathrm{S}_{1} \\ \mathrm{~S}_{2} \\ \mathrm{~S}_{3} \\ \mathrm{~S}_{4} \\ \mathrm{~S}_{5}\end{array}\right] \quad(37)$

Where:

$\mathrm{S}_{1}=\mathrm{U}_{\mathrm{t}} \mathrm{T}_{\mathrm{a}}+\alpha_{\mathrm{g}} \mathrm{I}$

$\mathrm{S}_{2}=-(2 \dot{\mathrm{m} C} / \mathrm{wL}) \mathrm{T}_{\mathrm{i}}$

$\mathrm{S}_{3}=\alpha_{\mathrm{p}} \tau_{\mathrm{g}} \mathrm{I}$

$\mathrm{S}_{4}=-\mathrm{S}_{2}$

$\mathrm{S}_{5}=-\mathrm{T}_{\mathrm{a}} \mathrm{U}_{\mathrm{b}}$

$\mathrm{S}_{6}=\mathrm{h}_{1}+\mathrm{h}_{\mathrm{rpg}}+\mathrm{U}_{\mathrm{t}}$

$\mathrm{S}_{7}=-\left[\mathrm{h}_{1}+\mathrm{h}_{2}+(2 \dot{\mathrm{m} C} / \mathrm{wL})\right]$

$\mathrm{S}_{8}=\mathrm{h}_{2}+\mathrm{h}_{3}+\mathrm{h}_{\mathrm{rpg}}+\mathrm{h}_{\mathrm{rpb}}+\frac{\mathrm{N}}{\mathrm{A}_{\mathrm{f}}}\left(2 \mathrm{kA}_{\mathrm{sf}} \mathrm{Lh}_{4}\right)^{1 / 2} \tanh \mathrm{MH}$

$\mathrm{S}_{9}=-\left[\mathrm{h}_{3}+\frac{\mathrm{N}}{\mathrm{A}_{\mathrm{f}}}\left(2 \mathrm{kA}_{\mathrm{sf}} \mathrm{Lh}_{4}\right)^{1 / 2} \tanh \mathrm{MH}\right]$

$\mathrm{S}_{10}=-\mathrm{S}_{9}$

$\mathrm{S}_{11}=-\left[\begin{array}{l}\mathrm{h}_{3}+\mathrm{h}_{5}+(2 \dot{\mathrm{mC}} / \mathrm{wL}) \\ +\frac{\mathrm{N}}{\mathrm{A}_{\mathrm{f}}}\left(2 \mathrm{kA}_{\mathrm{sf}} \mathrm{Lh}_{4}\right)^{1 / 2} \tanh \mathrm{MH}\end{array}\right]$

$\mathrm{S}_{12}=-\left(\mathrm{h}_{5}+\mathrm{h}_{\mathrm{rpb}}+\mathrm{U}_{\mathrm{b}}\right)$

$\mathrm{M}=\left(\frac{2 \mathrm{Lh}_{4}}{\mathrm{k}_{\mathrm{p}} \mathrm{A}_{\mathrm{sf}}}\right)^{1 / 2}$

For type III:

$$
\left[\begin{array}{ccccc}
\mathrm{S}_{6} & -\mathrm{h}_{1} & -\mathrm{h}_{\mathrm{rpg}} & 0 & 0 \\
\mathrm{~h}_{1} & \mathrm{~S}_{7} & \mathrm{~h}_{2} & 0 & 0 \\
-\mathrm{h}_{\mathrm{rpg}} & \mathrm{S}_{8} & \mathrm{~S}_{9} & \mathrm{~S}_{10} & -\mathrm{h}_{\mathrm{rpb}} \\
0 & 4 \dot{\mathrm{mC}} / \mathrm{wL} & \mathrm{S}_{11} & \mathrm{~S}_{12} & \mathrm{~h}_{6} \\
0 & 0 & \mathrm{~h}_{\mathrm{rpb}} & \mathrm{h}_{6} & \mathrm{~S}_{13}
\end{array}\right]\left[\begin{array}{c}
\mathrm{T}_{\mathrm{g}} \\
\mathrm{T}_{\mathrm{f} 1} \\
\mathrm{~T}_{\mathrm{p}} \\
\mathrm{T}_{\mathrm{f} 2} \\
\mathrm{~T}_{\mathrm{b}}
\end{array}\right]=\left[\begin{array}{c}
\mathrm{S}_{1} \\
\mathrm{~S}_{2} \\
\mathrm{~S}_{3} \\
\mathrm{~S}_{4} \\
\mathrm{~S}_{5}
\end{array}\right]
$$

where:

$$
\begin{aligned}
& \mathrm{S}_{1}=\mathrm{U}_{\mathrm{t}} \mathrm{T}_{\mathrm{a}}+\alpha_{\mathrm{g}} \mathrm{I} \\
& \mathrm{S}_{2}=-(2 \dot{\mathrm{m} C} / \mathrm{wL}) \mathrm{T}_{\mathrm{i}} \\
& \mathrm{S}_{3}=\alpha_{\mathrm{p}} \tau_{\mathrm{g}} \mathrm{I}
\end{aligned}
$$

$\mathrm{S}_{4}=-\mathrm{S}_{2}$

$\mathrm{S}_{5}=-\mathrm{T}_{\mathrm{a}} \mathrm{U}_{\mathrm{b}}$

$\mathrm{S}_{6}=\mathrm{h}_{1}+\mathrm{h}_{\mathrm{rpg}}+\mathrm{U}_{\mathrm{t}}$

$\mathrm{S}_{7}=-\left[\begin{array}{l}\mathrm{h}_{1}+\mathrm{h}_{2}+(2 \dot{\mathrm{mC}} / \mathrm{wL}) \\ +\frac{\mathrm{N}}{\mathrm{A}_{\mathrm{f}}}\left(2 \mathrm{kA}_{\mathrm{sf}} \mathrm{Lh}_{3}\right)^{1 / 2} \tanh \mathrm{MH}\end{array}\right]$

$\mathrm{S}_{8}=-\left[\mathrm{h}_{2}+\frac{\mathrm{N}}{\mathrm{A}_{\mathrm{f}}}\left(2 \mathrm{kA}_{\mathrm{sf}} \mathrm{Lh}_{3}\right)^{1 / 2} \tanh \mathrm{MH}\right]$

$\mathrm{S}_{9}=\mathrm{h}_{2}+\mathrm{h}_{4}+\mathrm{h}_{\mathrm{rpg}}+\mathrm{h}_{\mathrm{rpb}}+\frac{\mathrm{N}}{\mathrm{A}_{\mathrm{f}}}\left(2 \mathrm{kA}_{\mathrm{sf}} \mathrm{Lh}_{3}\right)^{1 / 2} \tanh \mathrm{MH}$

$+\frac{\mathrm{N}}{\mathrm{A}_{\mathrm{f}}}\left(2 \mathrm{kA}_{\mathrm{sf}} \operatorname{Lh}_{5}\right)^{1 / 2} \tanh \mathrm{MH}$

$\mathrm{S}_{10}=-\left[\mathrm{h}_{4}+\frac{\mathrm{N}}{\mathrm{A}_{\mathrm{f}}}\left(2 \mathrm{kA}_{\mathrm{sf}} \mathrm{Lh}_{5}\right)^{1 / 2} \tanh \mathrm{MH}\right]$

$\mathrm{S}_{11}=-\mathrm{S}_{10}$

$\mathrm{S}_{12}=-\left[\begin{array}{l}\mathrm{h}_{4}+\mathrm{h}_{6}+(2 \dot{\mathrm{mC}} / \mathrm{wL}) \\ +\frac{\mathrm{N}}{\mathrm{A}_{\mathrm{f}}}\left(2 \mathrm{kA}_{\mathrm{sf}} \mathrm{Lh}_{5}\right)^{1 / 2} \tanh \mathrm{MH}\end{array}\right]$

$\mathrm{S}_{13}=-\left(\mathrm{h}_{6}+\mathrm{h}_{\mathrm{rpb}}+\mathrm{U}_{\mathrm{b}}\right)$

$\mathrm{M}=\left(\frac{2 \mathrm{Lh}_{3,5}}{\mathrm{k}_{\mathrm{p}} \mathrm{A}_{\mathrm{sf}}}\right)^{1 / 2}$

The major design parameters are as follows: $\mathrm{L}=$ $2.4 \mathrm{~m}, \mathrm{w}=1 \mathrm{~m}, \alpha_{\mathrm{p}}=0.95, \alpha_{\mathrm{g}}=0.06, \varepsilon_{\mathrm{p}}=0.95, \varepsilon_{\mathrm{g}}=0.8$, $\tau_{\mathrm{g}}=0.9, \mathrm{U}_{\mathrm{b}}=1 \mathrm{~W} \mathrm{~m}^{-2} \mathrm{~K}, \mathrm{~V}=1 \mathrm{~m} \mathrm{~s}^{-2}, \mathrm{k}_{\mathrm{p}}=211 \mathrm{~W} \mathrm{mK}^{-1}$, $\mathrm{T}_{\mathrm{a}}=300 \mathrm{~K}, \mathrm{~T}_{\mathrm{i}}=303 \mathrm{~K}, \mathrm{I}=700 \mathrm{~W} \mathrm{~m}^{-2}, \mathrm{~d}=0.105 \mathrm{~m}$ and $\mathrm{w}=1 \mathrm{~m}$.

The mean air and wall temperatures of the first section are initially guessed and specified. In the study except that of the absorber which was set to a temperature $30^{\circ} \mathrm{C}$ above that of the ambient temperature. 
Incorporating these relations in Eq. $(2,4,7,9,12$, 14) and making some algebraic rearrangements, the mean temperature vector may be determined with Excel by matrix inversion form as:

$$
[\mathrm{T}]=[\mathrm{A}]^{-1}[\mathrm{~B}]
$$

The newly computed temperatures are then compared with the previously assumed ones and computed is repeated until all consecutive mean temperatures differ by less than $0.01{ }^{\circ} \mathrm{C}$. In the present case, a sufficient convergence for $T_{g}, T_{f 1}, T_{p}, T_{f 2}$ and $T_{b}$ are achieves in 4-6 iterations.

Physical properties: The physical properties of air are assumed to vary linearly with temperature ${ }^{\circ} \mathrm{C}$ (Alfegi et al., 2009).

Specific heat:

$$
\mathrm{C}_{\mathrm{p}}=1.0057+0.000066(\mathrm{~T}-27)
$$

Density:

$$
\rho=1.1774-0.00359(\mathrm{~T}-27)
$$

Therm:

$$
\mathrm{k}=0.02624+0.0000758(\mathrm{~T}-27)
$$

Al conductivity viscosity:

$$
\mu=\left[1.983+0.00184(\mathrm{~T}-27) 10^{-5}\right]
$$

Heat transfer coefficients: The heat transfer coefficients are computed accordingly, such as:

$$
\mathrm{h}_{\mathrm{w}}=2.8+3.3 \mathrm{~V}
$$

Where:

$\mathrm{h}_{\mathrm{w}}=$ Convection heat transfer coefficient due to wind $\mathrm{V}=$ Wind velocity

$$
\begin{aligned}
& \mathrm{h}_{\mathrm{rpg}}=\frac{\sigma\left(\mathrm{T}_{\mathrm{p}}^{2}+\mathrm{T}_{\mathrm{g}}^{2}\right)\left(\mathrm{T}_{\mathrm{p}}+\mathrm{T}_{\mathrm{g}}\right)}{\frac{1}{\varepsilon_{\mathrm{p}}}+\frac{1}{\varepsilon_{\mathrm{g}}}-1} \\
& \mathrm{~h}_{\mathrm{rgs}}=\frac{\sigma \varepsilon_{\mathrm{g}}\left(\mathrm{T}_{\mathrm{g}}+\mathrm{T}_{\mathrm{s}}\right)\left(\mathrm{T}_{\mathrm{g}}^{2}+\mathrm{T}_{\mathrm{s}}^{2}\right)\left(\mathrm{T}_{\mathrm{g}}-\mathrm{T}_{\mathrm{s}}\right)}{\mathrm{T}_{\mathrm{g}}-\mathrm{T}_{\mathrm{a}}}
\end{aligned}
$$

$$
\text { There } \mathrm{T}_{\mathrm{s}} \text { is the sky temperature: }
$$

$$
\begin{aligned}
& \mathrm{T}_{\mathrm{s}}=0.0552\left(\mathrm{~T}_{\mathrm{a}}\right)^{1.5} \\
& \mathrm{U}_{\mathrm{t}}=\left(\frac{1}{\mathrm{~h}_{\mathrm{w}}+\mathrm{h}_{\mathrm{rgg}}}\right)^{-1}
\end{aligned}
$$

The convective heat transfer coefficients are calculated using following relations:

$\mathrm{h}=\frac{\mathrm{k}}{\mathrm{D}_{\mathrm{h}}} \mathrm{Nu}$

Where:

$\mathrm{Nu}=$ Nusselt number

$D_{h}=$ the equivalence diameter of the channel

Nusselt number for laminar flow region $(\operatorname{Re}<2300)$, transition flow region $(2300<\operatorname{Re}<6000)$ and turbulent flow region respectively are (Bashria et al., 2007; Alfegi et al., 2009).

$\mathrm{Nu}=5.4+\frac{0.00190\left[\operatorname{Re} \operatorname{Pr}\left(\frac{\mathrm{D}_{\mathrm{h}}}{\mathrm{L}}\right)\right]^{1.71}}{1+0.00563\left[\operatorname{Re} \operatorname{Pr}\left(\frac{\mathrm{D}_{\mathrm{h}}}{\mathrm{L}}\right)\right]^{1.17}}$

$\mathrm{Nu}=0.116\left(\operatorname{Re}^{2 / 3}-125\right) \operatorname{Pr}^{1 / 3}\left[1+\left(\frac{D_{h}}{L}\right)^{2 / 3}\right]\left(\frac{\mu}{\mu_{w}}\right)^{0.14}$

$\mathrm{Nu}=0.018 \operatorname{Re}^{0.8} \operatorname{Pr}^{0.4}$

Where:

$\operatorname{Pr}=$ Prandtl

$\operatorname{Re}=$ The Reynolds number:

$$
\begin{aligned}
\operatorname{Re} & =\frac{\dot{\mathrm{m}} \mathrm{D}_{\mathrm{h}}}{\mathrm{A}_{\mathrm{f}} \mu} \\
\mathrm{D}_{\mathrm{h}} & =\frac{4 \mathrm{wd}}{2(\mathrm{w}+\mathrm{d})}
\end{aligned}
$$

\section{RESULTS AND DISCUSSION}

The useful gain by the solar collector to solar radiation with values of fluid inlet and outlet temperature and the fluid mass flow rate is given as follows:

$\mathrm{Q}_{\mathrm{u}}=\dot{\mathrm{mC}}\left(\mathrm{T}_{\mathrm{o}}-\mathrm{T}_{\mathrm{i}}\right)$ 
where, $\mathrm{C}$ is the specific heat of the fluid. The efficiency of the collector is given by:

$\eta=\frac{Q_{u}}{A_{f} I}=\frac{\dot{m} C\left(T_{o}-T_{i}\right)}{A_{f} I}$

Where:

$\mathrm{A}_{\mathrm{f}}=$ The area of collector

$\mathrm{I}=$ The solar radiation incident on the collector

Figure 4 shows the variation of the outlet temperature and efficiency with mass flow rate for different number of fins at type I.

Figure 5 shows the variation of the outlet temperature and efficiency with mass flow rate for different height of fins at type I.

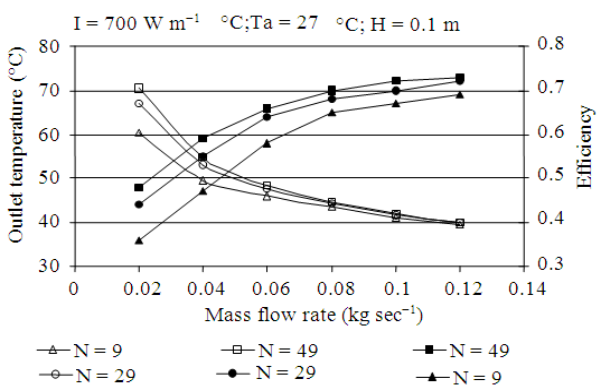

Fig. 4: Variation of outlet temperature and efficiency with mass flow rate for type I

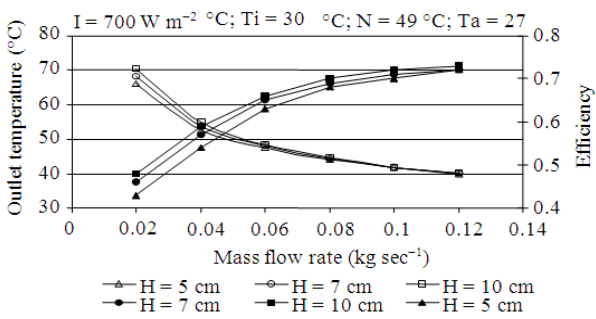

Fig. 5: Variation of outlet temperature and efficiency with mass flow rate for type I

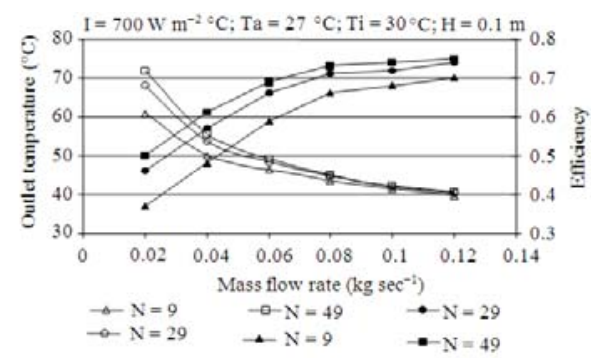

Fig. 6: Variation of outlet temperature and efficiency with mass flow rate for type II with different number of fins
Figure 6-7 show variation of the outlet temperature and efficiency with mass flow rate for type II.

Figure 8-9 show variation of the outlet temperature and efficiency with mass flow rate for type III.

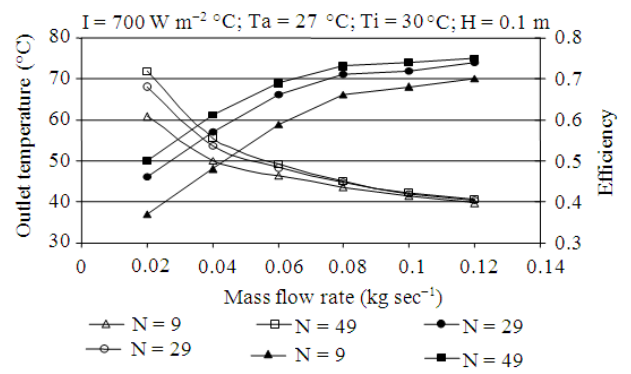

Fig. 7: Variation of outlet temperature and efficiency with mass flow rate for type II with different height of fins

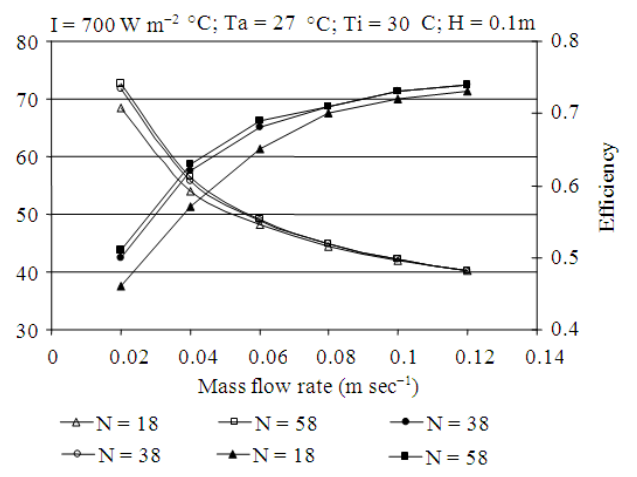

Fig. 8: Variation of outlet temperature and efficiency with mass flow rate for type III with different number of fins

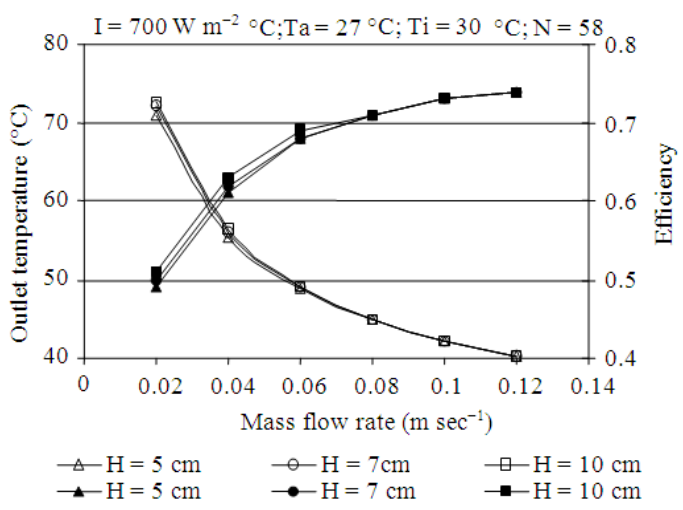

Fig. 9: Variation of outlet temperature and efficiency with mass flow rate for type III with different height of fins 
From the Fig. 4-9, it can be clearly seen that the efficiency is strongly dependent on the mass flow rate. The efficiencies of all type increased constantly up to $0.08 \mathrm{~kg} \mathrm{sec}^{-1}$ and then tended to approach a constant value. The collector efficiencies increase with flow rate, efficiency increase is about 30-35\%. On the other hand the effect of the number and height of fins on the efficiency of the solar collector is shown in Fig. 4-9. The efficiency increases with increasing the number and height of fins.

\section{CONCLUSION}

Performance curves of double-pass solar air collector with longitudinal fins absorber have been obtained. These include the effects of mass flow rate, number and height of fins on efficiency of the solar collector. The results show that the collector efficiency increases as the number and height of fins increases. The efficiency of the collector is strongly dependent on the flow rate. For a mass flow rate $0.02-0.1 \mathrm{~kg} \mathrm{sec}^{-1}$, efficiency increase is about $30 \%$. The double-pass solar collectors are efficiency about $36-73 \%$ in upper fins (type I), $37-75 \%$ in lower fins (type II) and $46-74 \%$ in upper and lower fins (type III).

\section{REFERENCES}

Alfegi, E.M.A., K. Sopian, M.Y.H. Othman and B.B. Yatim, 2009. Mathematical model of double pass photovoltaic thermal air collector with fins. Am. J. Environ. Sci., 5: 592-598. DOI: 10.3844/ajessp.2009.592.598

Bashria, A., A. Yousef, N.M. Adam, K. Sopian and A. Zaharim et al., 2007. Analysis of single and double passes v-grooves solar collector with and without porous media. Int. J. Energy Environ., 2: 109-114.
Dammak, N., B. Chaouachi, S. Gabsi and M. Bourouis, 2010. Optimization of the geometrical parameters of a solar bubble pump for absorption-diffusion cooling systems. Am. J. Eng. Applied Sci., 3: 693-698. DOI: 10.3844 /ajeassp.2010.693.698

Fudholi, A., K. Sopian, M.H. Ruslan, M.A. Alghoul and M.Y. Sulaiman, 2010. Review of solar dryers for agricultural and marine products. Renewable Sustainable Energy Rev., 14: 1-30. DOI: 10.1016/J.RSER.2009.07.032

Helal, O., B. Chaouachi, S. Gabsi and C. Bouden, 2010. Energetic performances study of an integrated collector storage solar water heater. Am. J. Eng. Applied Sci., 3: 152-158. DOI: 10.3844/ajeassp.2010.152.158

Ong, K.S., 1995. Thermal performance of solar air heaters: Mathematical model and solution procedure. Solar Energy., 55: 93-109. DOI: 10.1016/0038-092X(95)00021-I

Pradhapraj, M., V. Velmurugan and H. Sivarathinamoorthy, 2010. Review on porous and nonporous flat plate air collector with mirror enclosure. Int. J. Engineer. Sci. Technol., 2: 4013-4019.

Reda, S.M., 2010. Photostability of ponceau $2 \mathrm{r}$ doped thin film sol-gel silica as luminescent solar collector. Energy Res. J., 1: 36-41. DOI: 10.3844/erjsp.2010.36.41

Sopian, K., M.A. Alghoul, E.M. Alfegi, M.Y. Sulaiman and E.A. Musa, 2009. Evaluation of thermal efficiency of double-pass solar collector with porous-nonporous media. Renewable Energy., 34: 640-645. DOI: 10.1016/j.renene.2008.05.027 